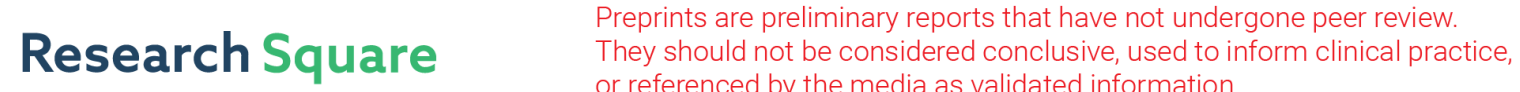 or referenced by the media as validated information. \\ Design and Analysis of a Novel Tree Climbing Robot Mechanism
}

\author{
Ru-Gui Wang ( $\nabla$ rugui@gxu.edu.cn ) \\ Guangxi Uninversity https://orcid.org/0000-0002-7351-3515 \\ Hai-Bo Huang \\ Guangxi Uninversity \\ Yi Li \\ Guangxi University \\ Ji-Wei Yuan \\ Nanjing University of Aeronautics and Astronautics
}

\section{Original Article}

Keywords: Tree climbing robot, kinematics, screw theory, singularity analysis

Posted Date: August 12th, 2020

DOI: https://doi.org/10.21203/rs.3.rs-55599/v1

License: (9) This work is licensed under a Creative Commons Attribution 4.0 International License. Read Full License 


\section{Title page}

\section{Design and Analysis of a Novel Tree Climbing Robot Mechanism}

Ru-Gui Wang, born in 1979, is currently a professor and a PhD candidate supervisor at Guangxi University, China. From 2015 to 2016 , he was a visiting scholar at the Centre for Robotics Research, King's College London, University of London, London, U.K. From 2019 to 2020, he was a visiting scholar at Tsinghua University, China. His research interests include robotic mechanisms, metamorphic mechanism, reconfigurable mechanisms, and design of robots.

E-mail: rugui@gxu.edu.cn

Hai-Bo Huang, born in 1995, is currently a master candidate at School of Mechanical Engineering, Guangxi University, China. His research interests include robot mechanism and multibody system dynamics.

E-mail: haibo.h@st.gxu.edu.cn

Yi Li, born in 1994, is currently a master candidate at School of Mechanical Engineering, Guangxi University, China. His research interests include robot mechanism and multibody system dynamics.

E-mail:1143461005@qq.com

Ji-Wei Yuan, born in 1993, is currently a PhD candidate at Nanjing University of Aeronautics and Astronautics, China. He received his bachelor degree from Guangxi University, China, in 2018. His research interests include bionics, biomechanics and multi-modal mobile robot.

E-mail: jiweiyuan@nuaa.edu.cn

\section{Corresponding author: Ru-Gui Wang E-mail: rugui@gxu.edu.cn}




\title{
Design and Analysis of a Novel Tree Climbing Robot Mechanism
}



\begin{abstract}
In this paper, a novel tree climbing robot mechanism was designed, based on the tree climbing movement and posture of the primates. The overall design and tree climbing gait of the tree climbing robot were analyzed in detail. According to the screw theory, the DOF of the leg of the tree climbing robot is calculated. The forward and inverse kinematics equations of the tree climbing robot were established and solved. The kinematics of the leg parallel mechanism was established, furthermore, the singularity of the leg mechanism was analyzed and three types of singularity were derived. The simplified diagrams and the corresponding model diagrams, at the singular points, were drawn. Finally, the movement is simulated and analyzed. And the changes of the leg joint angular and the foot-end displacement and the relationship between the driving displacement and angles of the tree climbing robot by numerical simulation is obtained at the same time. Prototype physical model of the tree climbing robot was made, which further verified the rationality and feasibility of the tree climbing robot mechanism studied in this paper.
\end{abstract}

Keywords: Tree climbing robot $\cdot$ kinematics - screw theory $\bullet$ singularity analysis

\section{Introduction}

Climbing robotics is a research field growing rapidly with primary focuses on the prototype design, development of climbing robots and applications. As the robotic technology constantly advances, climbing robots show promising application prospects in the fields of tree pruning [1], fruit picking [2] and high building cleaning [3-4]. Many scholars have carried out extensive research, in these application fields [5].

Ru-Gui Wang

rugui@gxu.edu.cn

1 School of Mechanical Engineering, Guangxi University, Nanning 530004, China

2 College of Mechanical and Electrical Engineering, Nanjing University of Aeronautics and Astronautics, Nanjing 210016, China
Climbing robots are divided into wall climbing robots [6-10] and tree climbing robots [11-14]. Most of the current tree-climbing robots are mainly wheeled, clawed and biomimetic [15], among which the wheeled tree climbing robot [16-21] mostly uses the ways of encircling to realize the climbing function of the tree climbing robot. The claw tree climbing [22-24] robot is a kind of tree climbing robot which uses the gripper or the claw to clamp the tree trunk and realize the tree climbing behavior, in vertical or handstand and other aspects of climbing a greater advantage. The biomimetic tree-climbing [25-30] robots can climb vertically or upside down. Most of these tree climbing robots have some characteristics, such as the wheeled tree-climbing robot is adapted to the trunk without branches, which cannot flexibly adapt to the actual working conditions, and the bionic tree-climbing robot has more advantages in vertical or upside-down climbing, while quadruped tree climbing robots, especially bionic robots, have not been investigated intensively.

Aiming at the shortage of mechanization and automation in forestry, agriculture and fruit picking, the tree climbing robot is further designed and studied. Compared with the existing wheeled, claw and bionic tree climbing robots, the quadruped tree climbing robot has the advantages of good stability and reliability, high obstacle surmounting ability and strong load capacity, it can be used in monitoring and testing, fruit picking and so on. The leg mechanism is an important guarantee for tree-climbing robot to move and perform tasks. Reasonable design and movement of the leg mechanism is an important premise. Therefore, it is necessary to further design and research the quadruped tree-climbing robot.

The quadruped tree-climbing robot mechanism, in this paper, is designed, based on the tree climbing action and posture of the primate. The rest of this paper is organized as follows. In Section II, the configuration design and trot gait analysis are described; Section III presents the DOF analysis of the leg mechanism by screw theory, and calculates the kinematics of the robot. Section IV analyzed the singularities and the kinematics of the leg parallel mechanism. Section V 
elaborates the numerical simulation of the process of tree climbing, joint angular and displacement change in a period time, and the relationship between the driving displacement and angles. Conclusions are given in Section VI.

\section{Mechanism Design of The Tree Climbing Robot}

\subsection{Design Inspiration}

As most often, the original inspiration comes from what surrounds us in nature. Observing primate climbing tree as shown in Figure 1, we can see the two legs of primates, staying basically at the same diagonal position, in course of climbing the tree, while the other two legs coordinate the climbing motion. In order to achieve coordinated movement of the robot legs, the waist will swing slightly according to the legs motion, and adapt to different trees. Besides, some scholars have studied the case of a waist with adaptable shape configuration [31].

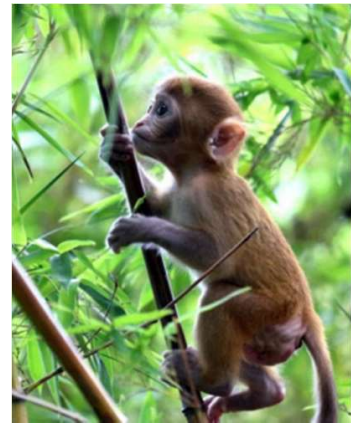

Figure 1 Example of primates climbing tree.

\subsection{Mechanism Design of Tree Climbing Robot}

Based on the tree-climbing movements and physical structure of primates in nature, observe and speculate on them, and then copy and imitate the mechanisms and functions of their systems, and combine the principles of bionics to carry out their mechanisms, functions and functions. Through research and application, the mechanism of a bionic tree-climbing robot was designed. The mechanism is composed of a frame and four legs, in which the leg mechanism design needs to meet the requirements as followings:

1) Realize the climbing action of holding and loosening of the trunk;

2) Realize the action of grasping and loosening of around the tree;

3) Realize the function of self-locking anti-falling.

Based on the above design analysis, the overall assembly diagram of the tree climbing robot as shown in Figure 2, in which the waist can swing slightly according to the legs motion.



Figure 2 Mechanism of the tree climbing robot.

Primates often climb, following a certain pattern and pace, during the process of climbing trees. This form of movement can be expressed in gait. The common gait of primates is diagonal gait, also known as trot gait, which is the gait of primates as they climb forward, accelerate or sprint, at a constant speed. As the two legs, on one diagonal line, step up and hold the tree, at the same time, the two legs, on the other diagonal line, are in a swing state, while remaining still. The gait diagram of the tree climbing motion are shown in Figure 3.

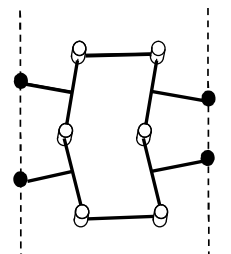

(a)

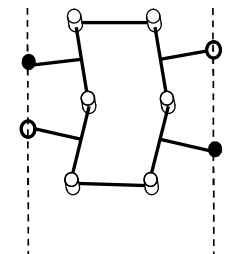

(b)

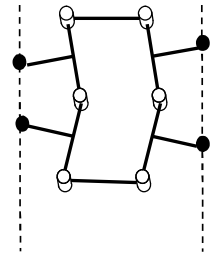

(c)

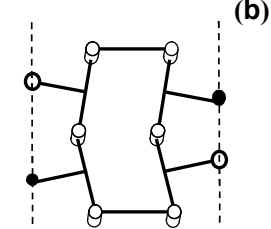

(d)



(e)
Figure 3 Diagram of tree climbing gait. (a) $t=0$. (b) $t=T / 4$. (c) $t=T / 2$. (d) $t=3 T / 4$. (e) $t=T$.

As shown in Figure 3, the black circle "•" means attached to the tree and the hollow circle "०" represents detached from the tree. The five phases (a)-(e) constitute a motion cycle of primates. During the tree climbing process, all four legs are held tight, like in state (a). With the first and third leg held up against the tree, while the second and fourth legs are held down against the tree, like in state (b). Again, in state (c), all four legs are held tight. With the second and fourth leg held up against the tree, while the first and third leg are down against the tree, like in state (d). When all four legs are held tight, it's back to the original state, like in state (e). In phases (b) and (d), the two legs in the diagonal position are in a hold-tight state, while the other two legs are in a swing-motion state. As shown in Figure 4 that Figure 4(a) corresponds to Figure 3(a), and F Figure 4(b) corresponds to Figure 3(b)and so on. The corresponding prototype physical model of the tree 
climbing robot is shown in Figure 5.

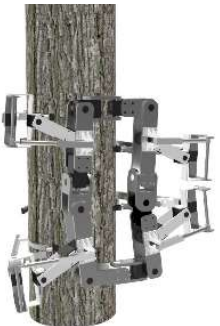

(a)

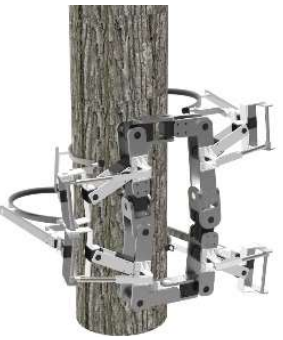

(b)

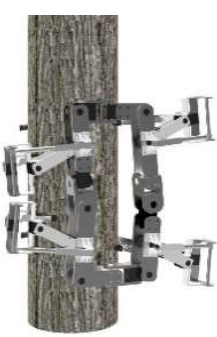

(c)

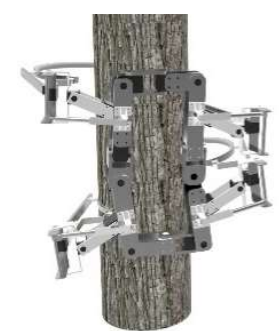

(d)

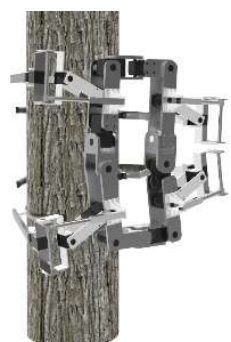

(e)
Figure 4 Tree climbing robot model in subsequent working states. (a) $t=0$. (b) $t=\mathrm{T} / 4$. (c) $t=\mathrm{T} / 2$. (d) $t=3 \mathrm{~T} / 4$. (e) $t=\mathrm{T}$.

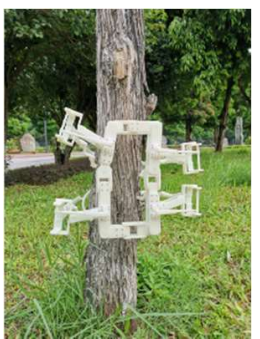

(a)

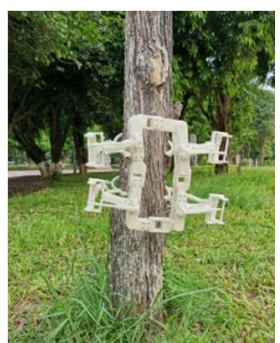

(b)

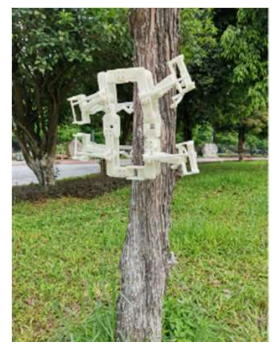

(c)

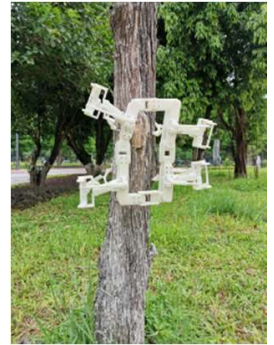

(d)

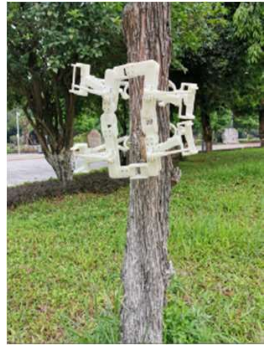

(e)
Figure 5 The prototype of the tree climbing robot mechanism: (a) the initial state. (b) Climbing the tree in $T / 2$. (c) Back to initial state in T. (d) Climbing the tree in 3T/2. (e) Hug the trunk in $2 \mathrm{~T}$

\section{Kinematics Analysis}

\subsection{The DOF of Leg Mechanism}

According to the tree-climbing robot mechanism, the diagram of the leg mechanism is designed as shown in Figure 6 .

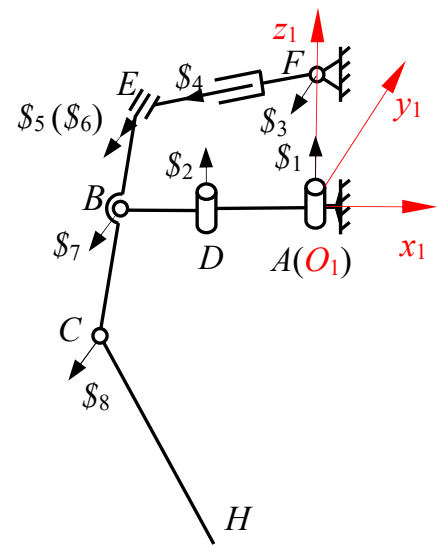

Figure 6 Leg mechanism link screw coordinate system

From the Figure 6, the leg mechanism is combined by series and parallel mechanism, in which the parallel mechanism is composed of closed-loop $A B D E F$, and the series mechanism is composed of open-loop $B C H$. The parallel mechanism also known as the hip joint mechanism, and the series mechanism consists of the self-locking mechanism and the foot-end.

Calculating the DOF of mechanism by screw theory, when established the coordinate system $O_{1}-x_{1} y_{1} z_{1}$, the screw system of the mechanism can be obtained by screw theory as follows:

$$
\begin{aligned}
& \$_{1}=[0,0,1,0,0,0]^{\mathrm{T}}, \quad \$_{2}=\left[0,0,1, d_{2}, e_{2}, 0\right]^{\mathrm{T}} \\
& \$_{3}=\left[0,1,0, d_{3}, 0, f_{3}\right]^{\mathrm{T}}, \$_{4}=\left[0,0,0, d_{4}, 0, f_{4}\right]^{\mathrm{T}} \\
& \$_{5}=\left[0,1,0, d_{5}, 0, f_{5}\right]^{\mathrm{T}}, \$_{6}=[0,0,0,0,1,0]^{\mathrm{T}} \\
& \$_{7}=\left[0,1,0, d_{7}, 0,0\right]^{\mathrm{T}}, \$_{8}=\left[0,1,0, d_{8}, 0, f_{8}\right]^{\mathrm{T}}
\end{aligned}
$$

From $\$_{i} \Delta \$_{i}^{r}=0$, the constraint spirals of the mechanism are found to be:

$$
\$_{1}^{r}=[0,0,0,1,0,0]^{\mathrm{T}}
$$

Thus, the mechanism has an anti-screw, namely, $\lambda_{0}=1$. Therefore, according to the modified G-K formula:

$$
M=d(n-g-1)+\sum_{i=1}^{g} f_{i}+v-\zeta=5 \times(7-7-1)+8+0-0=3
$$

Where, $M$ is the DOF, $d=6-\lambda_{0}$ represents the order, $n$ is the total number of the links, $g$ is the number of the kinematics pairs and $f_{i}$ represents the DOF of the number $g, \quad \zeta$ and $v$ is respectively the DOF of local and redundant of the mechanism.

\subsection{Forward Kinematics of Tree Climbing Robot}

The research of climbing robot motion includes kinematics and dynamics analysis [32], motion trajectory planning [33] and balance stability research [34]. 
We can know that each joint drive variable parameter solves the foot-end position, in a positive kinematics analysis of the tree climbing robot leg. The fixed coordinate system $O-x y z$ is attached on the tree climbing robot frame; the moving coordinate system $\sum_{A}$ is established at the joint $A$, while the moving coordinate system $\sum_{A}$ is translation along the $x$-axis of the fixed coordinate system $O-x y z$, as shown in Figure 7.

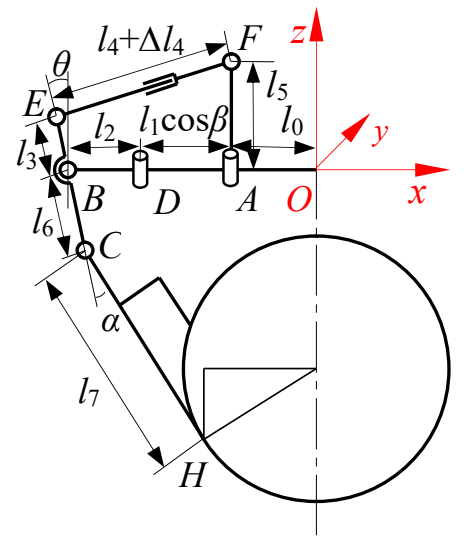

Figure 7 Leg mechanism coordinate system

In Figure 7, the length between the joint $A$ and the joint $B$ is $l_{1}$, while the angle of the joint $B$, swinging clockwise, under the force of the motor at the joint $A$, is $\beta$; under the force of the hydraulic cylinder, the moving coordinate system $\sum_{B}$ is inversely related to the moving coordinate system $\sum_{A}$ around the $y$-axis. The hour hand rotation angle is $\theta$, where the distance $E F$ is $l_{4}+\Delta l_{4} ; l_{2}, l_{7}$ and $l_{6}$ represent the distance between the joints $B D, H C$ and $B H$, respectively. The mechanism of leg, when climbing the tree, is shown in Fig. 7, where the following relations are clearly illustrated:

$$
{ }^{o} \boldsymbol{T}_{B}=\left[\begin{array}{cc}
{ }^{o} \boldsymbol{R}_{B} & { }^{o} \boldsymbol{P}_{B} \\
0 & 1
\end{array}\right]=\left[\begin{array}{cccc}
1 & 0 & 0 & -\left(l_{1} c \beta+l_{0}+l_{2}\right) \\
0 & 1 & 0 & -l_{1} s \beta \\
0 & 0 & 1 & 0 \\
0 & 0 & 0 & 1
\end{array}\right]
$$

Where, $\mathrm{c} \beta=\cos (\beta), s \beta=\sin (\beta) . l_{0}$ is the distance between the two mechanical legs, on the same side to the frame; Then,

$$
{ }^{b} \boldsymbol{T}_{H}=\left[\begin{array}{cc}
{ }^{b} \boldsymbol{R}_{H} & { }^{b} \boldsymbol{P}_{H} \\
0 & 1
\end{array}\right]=\left[\begin{array}{cccc}
c \theta \alpha & 0 & -s \theta \alpha & l_{7} s \theta \alpha+l_{6} s \theta \\
0 & 1 & 0 & 0 \\
s \theta \alpha & 0 & c \theta \alpha & -l_{7} c \theta \alpha-l_{6} c \theta \\
0 & 0 & 0 & 1
\end{array}\right]
$$

where, $c \theta \alpha=\cos (\theta+\alpha), s \theta \alpha=\sin (\theta+\alpha)$.

From Eq. (2) to Eq. (3), it is derived that:
${ }^{o} \boldsymbol{T}_{H}={ }^{o} \boldsymbol{T}_{B}{ }^{b} \boldsymbol{T}_{H}=\left[\begin{array}{cc}{ }^{o} \boldsymbol{R}_{H} & { }^{o} \boldsymbol{P}_{H} \\ 0 & 1\end{array}\right]=\left[\begin{array}{cccc}c \theta \alpha & 0 & -s \theta \alpha & A \\ 0 & 1 & 0 & -l_{1} s \beta \\ s \theta \alpha & 0 & c \theta \alpha-l_{7} c \theta \alpha-l_{6} c \theta \\ 0 & 0 & 0 & 1\end{array}\right]$

$A=l_{7} s \theta \alpha+l_{6} s \theta-\left(l_{1} c \beta+l_{0}+l_{2}\right)$

where,

$$
\begin{gathered}
{ }^{o} \boldsymbol{R}_{C}=\left[\begin{array}{ccc}
c \theta \alpha & 0 & -s \theta \alpha \\
0 & 1 & 0 \\
s \theta \alpha & 0 & c \theta \alpha
\end{array}\right] \\
{ }^{o} \boldsymbol{P}_{H}=\left[\begin{array}{c}
{ }^{o} x_{H} \\
{ }^{o} y_{H} \\
{ }^{o} z_{H}
\end{array}\right]=\left[\begin{array}{c}
l_{7} s \theta \alpha+l_{6} s \theta-\left(l_{1} c \beta+l_{0}+l_{2}\right) \\
-l_{1} s \beta \\
-l_{7} c \theta \alpha-l_{6} c \theta
\end{array}\right]
\end{gathered}
$$

\subsection{Inverse Kinematics of Tree Climbing Robot}

The inverse kinematics problem of the leg of a tree climbing robot is that the driving variable parameters of the joints are known to solve the joint position of the leg; that is, ${ }^{o} \boldsymbol{T}_{H}$ and ${ }^{\circ} \boldsymbol{T}_{A}$ of the known mechanical leg provide the joint variable parameters $\beta, \theta$ and $\alpha$

Assuming the foot-end position ${ }^{o} \boldsymbol{T}_{H}$ is:

$$
{ }^{o} \boldsymbol{T}_{H}=\left[\begin{array}{cccc}
n_{x} & o_{x} & a_{x} & { }^{o} x_{H} \\
n_{y} & o_{y} & a_{y} & { }^{o} y_{H} \\
n_{z} & o_{z} & a_{z} & { }^{o} z_{H} \\
0 & 0 & 0 & 1
\end{array}\right]={ }^{o} \boldsymbol{T}_{A}{ }^{a} \boldsymbol{T}_{B}{ }^{b} \boldsymbol{T}_{H}
$$

${ }^{o} \boldsymbol{T}_{A}^{-1}$ is multiplied by both left and right sides of Eq. (7), deriving:

$$
{ }^{o} \boldsymbol{T}_{A}^{-1{ }^{o}} \boldsymbol{T}_{H}={ }^{o} \boldsymbol{T}_{A}^{-1}{ }^{o} \boldsymbol{T}_{A}{ }^{a} \boldsymbol{T}_{B}{ }^{b} \boldsymbol{T}_{H}={ }^{a} \boldsymbol{T}_{H}
$$

Then,

$$
{ }^{o} \boldsymbol{T}_{A}^{-1 o}{ }^{o} \boldsymbol{T}_{H}=\left[\begin{array}{cccc}
n_{x} & o_{x} & a_{x} & { }^{o} x_{H}+l_{0} \\
n_{y} & o_{y} & a_{y} & { }^{o} y_{H} \\
n_{z} & o_{z} & a_{z} & { }^{o} z_{H} \\
0 & 0 & 0 & 1
\end{array}\right]
$$

And then,

$$
{ }^{a} \boldsymbol{T}_{H}=\left[\begin{array}{cccc}
c \theta \alpha & 0 & -s \theta \alpha & l_{7} s \theta \alpha+l_{6} s \theta-\left(l_{1} c \beta+l_{2}+l_{0}\right) \\
0 & 1 & 0 & -l_{1} s \beta \\
s \theta \alpha & 0 & c \theta \alpha & l_{7} c \theta \alpha-l_{6} c \theta \\
0 & 0 & 0 & 1
\end{array}\right]
$$

The corresponding terms on both sides of Eq. (8) are equal, so it follows that: 


$$
\left\{\begin{array}{l}
\beta=-\operatorname{asin}\left({ }^{o} y_{H} / l_{2}\right) \\
\theta=\operatorname{atan} 2\left(K, \pm \sqrt{d_{2}^{2}+d_{1}^{2}-K^{2}}\right)-\operatorname{atan} 2\left(d_{2}, d_{1}\right) \\
\alpha=\operatorname{atan} 2\left(M, \pm \sqrt{d_{2}^{2}+d_{1}^{2}-M^{2}}\right)-\operatorname{atan} 2\left(K, \pm \sqrt{d_{2}^{2}+d_{1}^{2}-K^{2}}\right)
\end{array}\right.
$$

where,

$$
\left\{\begin{array}{l}
d_{1}={ }^{o} y_{H}+2 l_{0} l_{1} c \beta, d_{2}=-{ }^{o} z_{H} \\
a_{1}=l_{7}, a_{2}=l_{6} \\
K=\frac{d_{1}^{2}+d_{2}^{2}+a_{1}^{2}-a_{2}^{2}}{2 a_{2}} \\
M=\frac{d_{1}^{2}+d_{2}^{2}+a_{2}^{2}-a_{1}^{2}}{2 a_{1}}
\end{array}\right.
$$

The two solutions corresponding to the positive and negative signs in Eq. (11) are all possible solutions, thus $\theta$ has two possible solutions, and $\alpha$ has four possible solutions.

\section{Singularity Analysis}

\subsection{Forward Kinematics}

As shown in Figure 8 that the projection of the parallel mechanism $A B D E F$. The lengths of the links $A F, A D, A B$ and $B E$ are $l_{5}, l_{1}, l_{2}$ and $l_{3}$, respectively, while the initial length of the link $E F$ is $l_{4}+\Delta l_{4}$, the rotation angle of the link $B E$, relative to the $y$-axis, is $\theta$. The input parameters of the mechanism are the connecting rod $A D$ rotation angle $\beta$ and the connecting rod $E F$ elongation $\Delta l_{4}$.

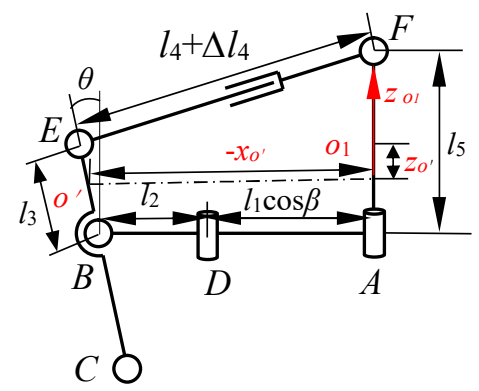

Figure 8 Projection diagram of parallel mechanism $A B D E F$

$\boldsymbol{P}, \boldsymbol{R}$ denote the position and attitude of the moving platform center $O^{\prime}$ relative to the fixed platform, described by:

$$
\begin{gathered}
\boldsymbol{P}=\left[x_{O^{\prime}}, y_{O^{\prime}}, z_{O^{\prime}}\right]^{\mathrm{T}} \\
\boldsymbol{R}=\left[\begin{array}{ccc}
\cos \theta & 0 & \sin \theta \\
0 & 1 & 0 \\
-\sin \theta & 0 & \cos \theta
\end{array}\right]
\end{gathered}
$$

The coordinates of $A, F, B$, and $E$, in the fixed coordinate system, can be given by:

$$
\left\{\begin{array}{l}
(F)_{O}=\left[0,0,0.5 l_{5}\right]^{\mathrm{T}} \\
(A)_{O}=\left[0,0,-0.5 l_{5}\right]^{\mathrm{T}} \\
(B)_{O}=\left[-x_{O^{\prime}}+0.5 l_{3} \sin \theta, y_{O^{\prime}},-z_{O^{\prime}}-0.5 l_{3} \cos \theta\right]^{\mathrm{T}} \\
(E)_{O}=\left[-x_{O^{\prime}}-0.5 l_{3} \sin \theta, y_{O^{\prime}},-z_{O^{\prime}}+0.5 l_{3} \cos \theta\right]^{\mathrm{T}}
\end{array}\right.
$$

As shown in Fig.8, the following geometric relations are satisfied:

$$
\left\{\begin{array}{l}
x_{O^{\prime}}=l_{1} \cos \beta+l_{2}+0.5 l_{3} \sin \theta \\
y_{O^{\prime}}=l_{1} \sin \beta \\
z_{O^{\prime}}=0.5 l_{3} \cos \theta-0.5 l_{5}
\end{array}\right.
$$

And

$$
|E F|^{2}=\left(z_{O^{\prime}}+0.5 l_{3} \cos \theta-0.5 l_{5}\right)^{2}+\left(x_{O^{\prime}}+0.5 l_{3} \sin \theta\right)^{2}=\left(l_{4}+\Delta_{4}\right)^{2}
$$

From Eq. (16) to Eq. (17) it is derived that:

$$
\left(l_{5}-l_{3} \cos \theta\right)^{2}+\left(l_{1} \cos \beta+l_{2}+l_{3} \sin \theta\right)^{2}=\left(l_{4}+\Delta l_{4}\right)^{2}
$$

Let $t=\tan \left(\frac{\theta}{2}\right)$, then:

$$
t=\frac{-c \pm \sqrt{b^{2}+c^{2}-a^{2}}}{a+b}
$$

where, $\quad a=\left(l_{1} \cos \beta+l_{2}\right)^{2}+l_{3}^{2}+l_{5}^{2}-\left(l_{4}+\Delta l_{4}\right)^{2}$

$b=2 l_{3} l_{5}, c=2 l_{3}\left(l_{1} \cos \beta+l_{2}\right)$.

So, it follows that:

$$
\left\{\begin{array}{l}
x_{O^{\prime}}=l_{2} \cos \beta+0.5 l_{3} \sin (2 \arctan (t)) \\
y_{O^{\prime}}=l_{2} \sin \beta \\
z_{O^{\prime}}=0.5 l_{3} \cos (2 \arctan (t))-0.5 l_{1}
\end{array}\right.
$$

Obviously, for each set of input parameters $\beta$ and $\Delta l_{4}$, the kinematic positive solution has four sets of solutions.

\subsection{Singularity Analysis}

The singularity analysis of the parallel mechanism (Figure 8 ) is carried out. The output parameters are set to $\theta, y_{o}$, so it is derived that:

$$
\boldsymbol{A}\left[\begin{array}{c}
\dot{\theta} \\
\dot{y}_{O^{\prime}}
\end{array}\right]=\boldsymbol{B}\left[\begin{array}{c}
\dot{\beta} \\
\Delta \dot{l}_{4}
\end{array}\right]
$$

The Jacobian matrix of parallel mechanism can be calculated by:

$$
\boldsymbol{J}=\boldsymbol{A}^{-1} \boldsymbol{B}
$$

Where,

$$
\begin{aligned}
& \boldsymbol{A}=\left[\begin{array}{ll}
K & 0 \\
0 & 1
\end{array}\right], \boldsymbol{B}=\left[\begin{array}{cc}
H & F \\
l_{2} \cos \beta & 0
\end{array}\right] \\
& K=\left(1+t^{2}\right)(a+b)^{2} \sqrt{d^{2}-a^{2}} \\
& F=-4\left(l_{4}+\Delta l_{4}\right)\left(4 \sqrt{d^{2}-a^{2}} \pm\left(d^{2}+a b\right)\right)
\end{aligned}
$$

The value of $a$ does not affect the matrix determinant.

We can distinguish three kinds of singularities 
concerning the parallel mechanism [35-36].

(I) when $\operatorname{det}(\boldsymbol{A})=0$ and $\operatorname{det}(\boldsymbol{B}) \neq 0$, as well as $d^{2}=a^{2}$, so it is derived that:

$$
\Delta l_{4}=\sqrt{l_{5}^{2}+l_{2}^{2}+l_{3}^{2}+l_{1}^{2} \cos ^{2} \beta+2 l_{1} l_{2} \cos \beta+\sqrt{b^{2}+c^{2}}}-l_{4}
$$

Or

$$
\Delta l_{4}=\sqrt{l_{5}^{2}+l_{2}^{2}+l_{3}^{2}+l_{1}^{2} \cos ^{2} \beta+2 l_{1} l_{2} \cos \beta-\sqrt{b^{2}+c^{2}}}-l_{4}
$$

(II) when $\operatorname{det}(\boldsymbol{A}) \neq 0$ and $\operatorname{det}(\boldsymbol{B})=0$, it is derived that:

$$
\Delta l_{4}=-l_{4}
$$

(III) when $\operatorname{det}(\boldsymbol{A})=0$ and $\operatorname{det}(\boldsymbol{B})=0$, it is derived that:

$$
\Delta l_{4}=\sqrt{\left(l_{5}+l_{3}\right)^{2}+\left(l_{1} \cos \beta+l_{2}\right)^{2}}-l_{4}
$$

Figure 9- Figure 12 shows the projection diagram of the parallel mechanism, in the cases of the three singularities.

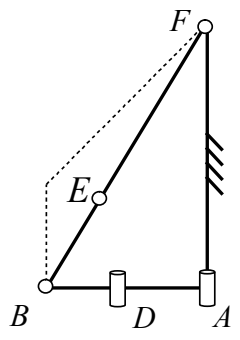

(a)

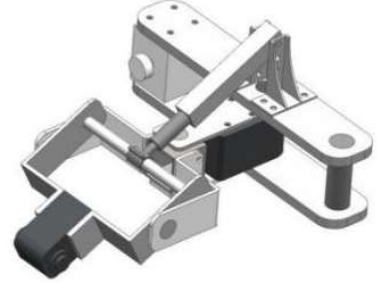

(b)
Figure 9 Singularity (I): The cases of the Eq. (23). (a) Diagram of the parallel mechanism. (b) Model of the parallel mechanism.

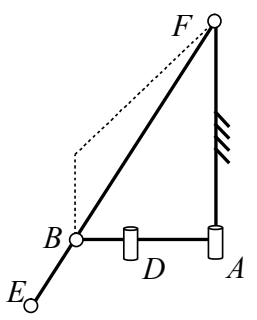

(a)

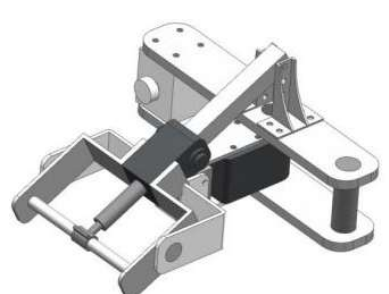

(b)
Figure 10 Singularity (II): The cases of the Eq. (24). (a) Diagram of the parallel mechanism. (b) Model of the parallel mechanism.

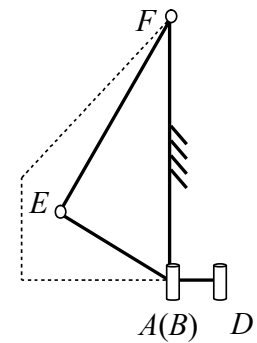

(a)

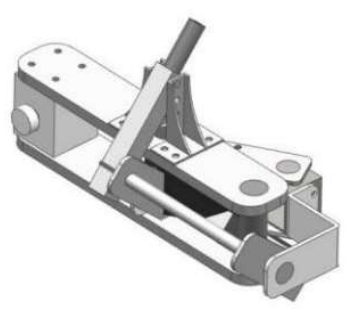

(b)
Figure 11 Singularity (II): The cases of the Eq. (25). (a) Diagram of the parallel mechanism. (b) Model of the parallel mechanism.

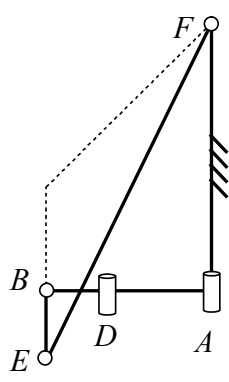

(a)

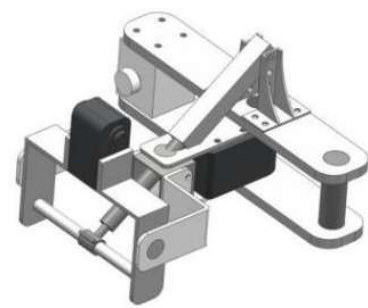

(b)
Figure 12 Singularity (III): The cases of the Eq. (26). (a) Diagram of the parallel mechanism. (b) Model of the parallel mechanism.

The singularity analysis produces the relation between the parameters of the mechanism in the singular position. This is a strong tool, serving the mechanism, to avoid the singular position.

\section{Numerical Simulation}

\subsection{The Process of Angular Change Period Time}

In order to verify the correctness of the change curve of tree climbing robot of the leg joint angular in the period time, we have: $0 \leq \beta \leq 22^{\circ},-30^{\circ} \leq \theta \leq 15^{\circ},-45^{\circ} \leq \alpha \leq 30^{\circ}$.

the numerical calculation is used to simulate and analysis the angular as shown in Figure 13.

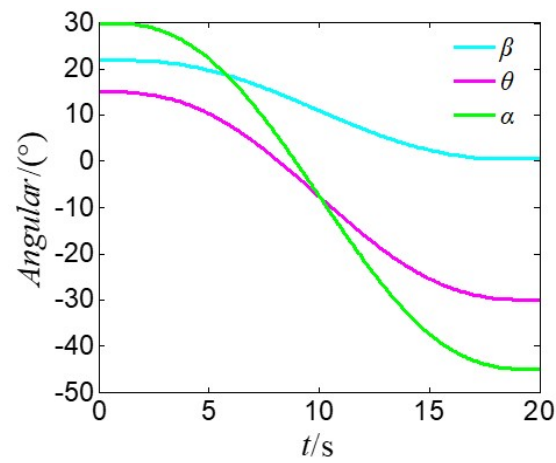

Figure 13 The change curve of the leg joint angular over a time period.

In Figure 13 that the angular $\beta, \theta$ and $\alpha$ shows parabolic curve from minimum to maximum, in which the curve is smooth and flexible. The direction of angle $\theta$ and $\alpha$ changes when $t=10 \mathrm{~s}$ and reaches the $\max$ at $20 \mathrm{~s}$. The angle $\beta$ gradually decreases with zero at $20 \mathrm{~s}$, indicating that the tree climbing robot is in moving forward in period.

\subsection{The Process of Displacement Change Period Time}

In order to verify the correctness of the above forward kinematics of tree climbing robot, we have: $\mathrm{m}=80 \mathrm{~mm}$, $l_{0}=40 \mathrm{~mm}, \quad l_{1}=85 \mathrm{~mm}, \quad l_{2}=40 \mathrm{~mm}, \quad l_{3}=20 \mathrm{~mm}, \quad l_{6}=43 \mathrm{~mm}$, $l_{7}=225 \mathrm{~mm}$. Simulate and analysis the displacement as shown in Figure 14. 




Figure 14 The change curve of the displacement over a time period.

It can be seen from Figure 14 that the displacement of $x$-axis gradually changes from negative to positive value with time, which indicates that the foot-end moves away from the fixed coordinate center. It has reached the limit position at $t=20 \mathrm{~s}$. The displacement of $y$-axis changes increases with time, indicating that the leg is in a climbing state. Besides, the displacement of $z$-axis is a parabolic curve, in which at $t=20 \mathrm{~s}$, the displacement reaches the max.

\subsection{Drive Displacement Calculation}

Calculation and simulation of drive displacement, we have: $l_{1}=85 \mathrm{~mm}, \quad l_{2}=40 \mathrm{~mm}, \quad l_{3}=20 \mathrm{~mm}, \quad l_{4}=55 \mathrm{~mm}, \quad l_{5}=48 \mathrm{~mm}$, $-22^{\circ} \leq \beta \leq 22^{\circ},-30^{\circ} \leq \theta \leq 30^{\circ}$.

Combining equation (18), the relationship between the driving displacement $\Delta l_{4}$ and the angles $\beta$ and $\theta$ can be obtained as shown in Figure 15. When the angle $\beta$ is fixed, the driving displacement $\Delta l_{4}$ and the angle $\theta$ show a linear change, indicating that the electric push rod will cause an angle change when driving; when the angle $\theta$ is constant, the driving displacement $\Delta l_{4}$ and the angle $\beta$ show a parabolic change, indicating that the tree climbing robot is on the leg When the tree is climbed in the forward and reverse directions of the motor, the legs can automatically hold the trunk while swinging; where, when $\beta=0^{\circ}$, the value range of the drive displacement $\Delta l_{4}$ is: $[63 \mathrm{~mm}$, $84 \mathrm{~mm}]$.

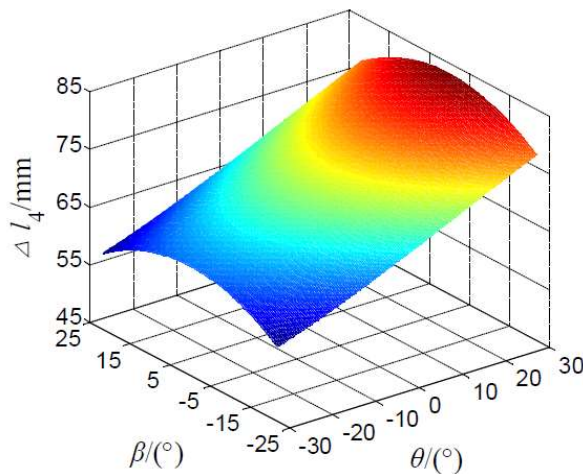

Figure 15 Relationship between the driving displacement $\Delta l_{4}$ and value range of angle $\beta$ and $\theta$.

\section{Conclusions}

Inspired by the primates climbing trees, and based on the tree climbing movement and posture of the primate, a quadruped tree climbing robot is designed, in which the robot can flexibly imitate the tree climbing movement and posture of the primate. First, according to the design requirements, the whole mechanism of the climbing robot is designed, and the gait of the tree climbing robot is briefly explained and imitated. Second, the DOF of the leg of the tree climbing robot is calculated by using the screw theory. Then, the mathematical model of forward and inverse kinematics equations of the tree climbing robot is built and solved, and as well as the kinematics of the parallel mechanism $A B D E F$. Besides, analyzing the singularity of the parallel mechanism $A B D E F$, and obtaining three types singularity, while the simplified diagrams and the corresponding model diagrams, at the singular points, are illustrated. Finally, in order to understand the movement of the tree climbing robot intuitively, the movement is simulated and analyzed and the changes of the leg joint angular and the foot-end displacement and the relationship between the driving displacement and angles of the tree climbing robot by simulation software is obtained at the same time. The tree-climbing robot mechanism can be used in areas such as tree pruning, fruit picking and collecting specimens, military monitoring and rod-shaped building cleaning and maintenance, quality inspection and other application fields.

Future work: Based on the contributions of this paper, the further research is mainly about the following parts:

1) The working principle of the primate crest column is a combination of movements, presenting a system with a high-degree-of-freedom, with multiple coordinated movements for climbing trees and performing tasks in the future, it is necessary to further the control analysis.

2) There's no doubt that the trajectory planning of leg movements needs to be studied further, and it's an important foundation for tree climbing robots to overcome obstacles and perform tasks.

\section{Declaration}

\section{Acknowledgements}

The authors thank CCMMS2020 for the recommendation and many helpful comments to improve the article.

\section{Funding}

Supported by National Natural Science Foundation of China (Grant No. 51865001).

\section{Availability of data and materials}

The datasets supporting the conclusions of this article are included within the article. 


\section{Authors' contributions}

RGW was in charge of the whole trial; $\mathrm{HBH}$ and YL wrote the manuscript; JWY were in charge of the prototype manufacturing and data analysis. All authors read and approved the final manuscript.

\section{Competing interests}

The authors declare no competing financial interests.

\section{Consent for publication}

Not applicable

\section{Ethics approval and consent to participate}

Not applicable

\section{References}

[1] $\mathrm{Y}$ Ishigure, $\mathrm{K}$ Hirai, $\mathrm{H}$ Kawasaki. A pruning robot with a power-saving chainsaw drive. Proceedings of IEEE International Conference on Mechatronics and Automation, Takamatsu, Japan, August 4-7, 2013: 1223-1228.

[2] T S Wibowo, I A Sulistijono, A Risnumawan. End-to-end coconut harvesting robot. Proceedings of International Electronics Symposium, Denpasar, Indonesia, September 29-30, 2016: 444-449.

[3] W Myeong, H Myung. Development of a Wall-Climbing Drone Capable of Vertical Soft Landing Using a Tilt-Rotor Mechanism. IEEE Access, 2019, 7: 4868-4879.

[4] X S Gao, K Kikuchi. Study on a kind of wall cleaning robot. Proceedings of International Conference on Robotics and Biomimetics, Shenyang, China, August 22-26, 2004: 391-394.

[5] D Schmidt, $\mathrm{K}$ Berns. Climbing robots for maintenance and inspections of vertical structures-A survey of design aspects and technologies. Robotics and Autonomous Systems, 2013, 61(12): 1288-1305.

[6] F Gao, J Fan, L Zhang, et al. Magnetic crawler climbing detection robot basing on metal magnetic memory testing technology. Robotics and Autonomous Systems, 2020, 125: 103439.

[7] Y Liu, B Lim, J W Lee, et al. Steerable dry-adhesive linkage-type wall-climbing robot. Mechanism and Machine Theory, 2020, 153: 103987.

[8] J Shen, Y Liu. Design and Analysis of an Obstacle-crossing Wall-climbing Robot Mechanism. Proceedings of Annual International Conference on Cyber Technology in Automation Control and Intelligent Systems, Shenyang, China, June 8-12, 2015 : 2067-2072.

[9] W C Myeong, K Y Jung, S W Jung, et al. Development of a drone-type wall-sticking and climbing robot. Proceedings of $12 \mathrm{th}$ International Conference on Ubiquitous Robots and Ambient Intelligence, Goyang, South Korea, October 28-30, 2015: 386-389.

[10] K Huang, Y Sun, J Yang, et al. Researches on a wall-climbing robot based on electromagnetic adsorption. Proceedings of $3 \mathrm{rd}$ Information Technology, Networking, Electronic and Automation Control Conference, Chengdu, China, March 15-17, 2019: 644-647.

[11] M I Nor Faizal, W A F W Othman, S S N A Syed Hassan. Development of pole-like tree climbing robot. Proceedings of International Conference on Control System, Computing and Engineering, George Town, Malaysia, November 27-29, 2015: 224-229.

[12] M A Mustapa, W A F W Othman, E A Bakar, et al. Development of Pole-Like Tree Spiral Climbing Robot[M]. 2018, 285-293.
[13] B Sebastian, D Ray, S Majumder. Design and analysis of a tree climbing robot. Proceedings of Conference on Advances In Robotics, Goa, India, July, 2015: 1-7.

[14] D Ren, S Yang, G Yan, et al. Study on a Novel Wheel Type Tree-climbing Robot. Proceedings of International Symposium on Computational Intelligence and Design, Hangzhou, China, December 13-14, 2014: 150-153.

[15] J Yuan, R Wang. Review of research on tree-climbing robot. Machine Design and Research, 2018, 34(06): 10-15. (in Chinese)

[16] P Gui, L Tang, S Mukhopadhyay. A novel design of anti-falling mechanism for tree pruning robot. Proceedings of 10th Conference on Industrial Electronics and Applications, Auckland, New Zealand, June 15-17, 2015: 812-816.

[17] P Gui, L Tang, S Mukhopadhyay. Anti-falling Tree Climbing Mechanism Optimization. Proceedings of 2nd Asia-Pacific Conference on Intelligent Robot Systems, Wuhan, China, June 16-18, 2017: 284-288.

[18] P Gui, L Tang, S Mukhopadhyay. Tree pruning robot tilting control using fuzzy logic. Proceedings of Eleventh International Conference on Sensing Technology, Sydney, NSW, Australia, December 4-6, 2017: 1-5.

[19] P Gui, L Tang, S Mukhopadhyay. A Novel Robotic Tree Climbing Mechanism With Anti-Falling Functionality for Tree Pruning. Journal of Mechanisms and Robotics, 2018, 10: 145021.

[20] $\mathrm{G} \mathrm{Fu}, \mathrm{X} \mathrm{Liu}, \mathrm{Y}$ Chen, et al. Fast-growing forest pruning robot structure design and climbing control. Advances in Manufacturing, 2015, 3(2): 166-172.

[21] Y Li, M Z Q Chen, Y H Chen, et al. Design of a one-motor tree-climbing robot. Proceedings of International Conference on Information and Automation, Lijiang, China, August 8-10, 2015: 26-31.

[22] Y Liu, T L Lam, H Qian, et al. Design and analysis of gripper with retractable spine for tree climbing robots. Proceedings of International Conference on Information and Automation, Hailar, China, July 28-30, 2014: 350-355.

[23] T L Lam, Y Xu. Climbing Strategy for a Flexible Tree Climbing Robot-Treebot. IEEE Transactions on Robotics, 2011, 27(6): 1107-1117.

[24] T L Lam, Y Xu. Mechanical design of a tree gripper for miniature tree-climbing robots. Proceedings of International Conference on Intelligent Robots and Systems, San Francisco, CA, USA, September 25-30, 2011: 1487-1492.

[25] R de Bernardi, J J D Cruz. Kamanbaré: A tree-climbing biomimetic robotic platform for environmental research[M]. 2007.

[26] L Jiang, Y Guan, C Cai, et al. Gait Analysis of a Novel Biomimetic Climbing Robot. Journal of Mechanical Engineering, 2010, 46(15): 17-22. (in Chinese)

[27] S Kim, M Spenko, S Trujillo, et al. Smooth Vertical Surface Climbing With Directional Adhesion. IEEE transactions on robotics, 2008, 24(1): 65-74

[28] Y Guan, H Zhu, W Wu, et al. A Modular Biped Wall-Climbing Robot With High Mobility and Manipulating Function. IEEE/ASME Transactions on Mechatronics, 2013, 18(6): 1787-1798.

[29] H Zhu, Y Guan, W Wu, et al. Autonomous Pose Detection and Alignment of Suction Modules of a Biped Wall-Climbing Robot. IEEE/ASME Transactions on Mechatronics, 2015, 20(2): 653-662.

[30] W Chen, S Gu, L Zhu, et al. Representation of truss-style structures for autonomous climbing of biped pole-climbing robots. Robotics and Autonomous Systems, 2018, 101: 126-137.

[31] W Zhen, X Kang, X Zhang, et al. Gait Planning of a Novel Metamorphic Quadruped Robot. Journal of Mechanical Engineering, 2016, 52(11): 26-33. (in Chinese)

[32] K Ba, B Yu, Z Gao, et al. Dynamic Compliance Analysis for LHDS of Legged Robot, Part B: Force-Based Impedance Control. IEEE Access, 2018, 6: 74799-74811.

[33] M Elbanhawi, M Simic. Sampling-Based Robot Motion Planning: A Review. IEEE Access, 2014, 2: 56-77.

[34] J Lin, K Hwang. Balancing and Reconstruction of Segmented Postures for Humanoid Robots in Imitation of Motion. IEEE Access, 2017, 5: 17534-17542.

[35] C Gosselin, J Angeles. Singularity Analysis of Closed-Loop 
Kinematic Chains. IEEE transactions on robotics and automation, 1990, 6(3): 281-290.

[36] X Liu, C Wu, J Wang. A New Approach for Singularity Analysis and Closeness Measurement to Singularities of Parallel Manipulators. Journal of Mechanisms and Robotics, 2012, 4: 410014

\section{Biographical notes}

Ru-Gui Wang, born in 1979, is currently a professor and a $\mathrm{PhD}$ candidate supervisor at Guangxi University, China. From 2015 to 2016, he was a visiting scholar at the Centre for Robotics Research, King's College London, University of London, London, U.K. From 2019 to 2020, he was a visiting scholar at Tsinghua University, China. His research interests include robotic mechanisms, metamorphic mechanism, reconfigurable mechanisms, and design of robots.

E-mail: rugui@gxu.edu.cn

Hai-Bo Huang, born in 1995, is currently a master candidate at School of Mechanical Engineering, Guangxi University, China. His research interests include robot mechanism and multibody system dynamics.

E-mail: haibo.h@st.gxu.edu.cn

Yi Li, born in 1994, is currently a master candidate at School of Mechanical Engineering, Guangxi University, China. His research interests include robot mechanism and multibody system dynamics.

E-mail: 1143461005@qq.com

Ji-Wei Yuan, born in 1993, is currently a PhD candidate at Nanjing University of Aeronautics and Astronautics, China. He received his bachelor degree from Guangxi University, China, in 2018. His research interests include bionics, biomechanics and multi-modal mobile robot.

E-mail: jiweiyuan@nuaa.edu.cn 


\section{Figures}

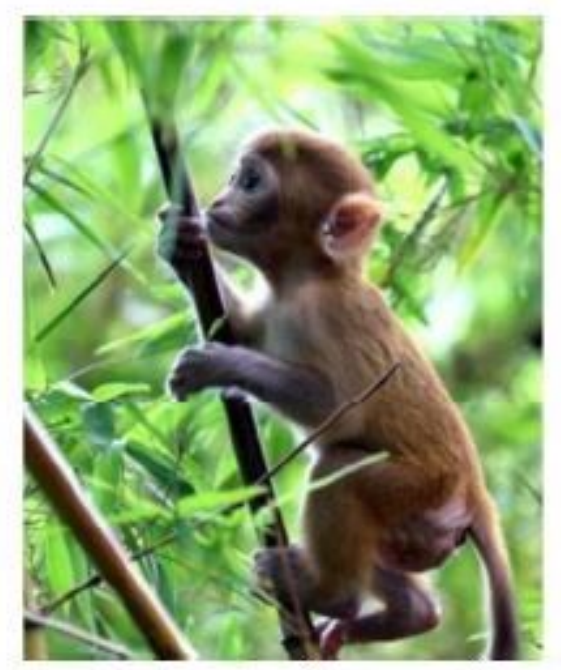

\section{Figure 1}

Example of primates climbing tree.

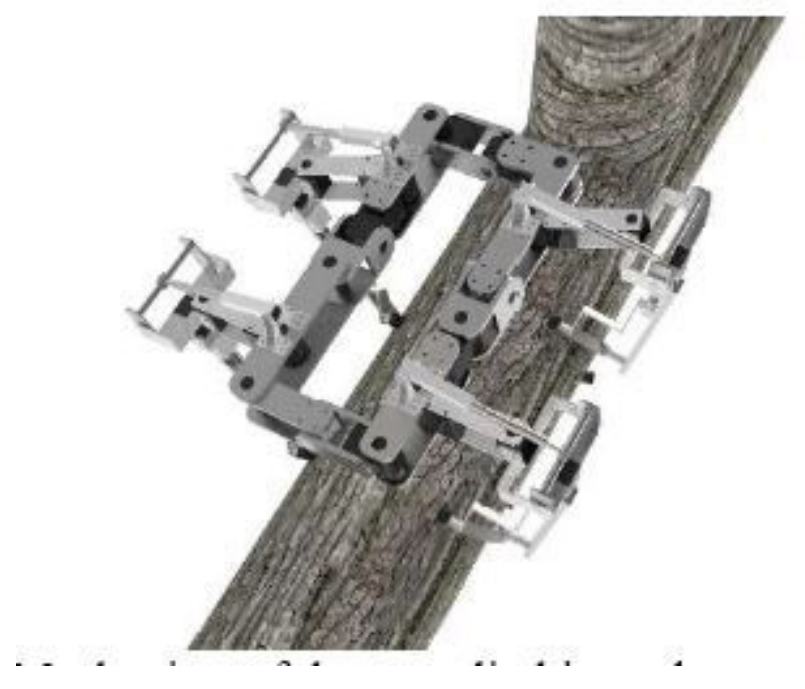

Figure 2

Mechanism of the tree climbing robot. 


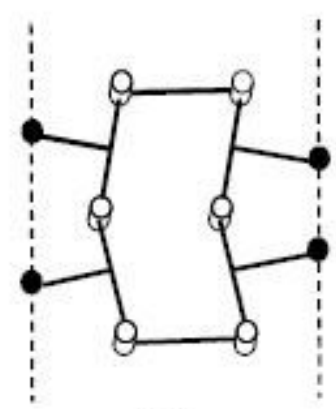

(a)

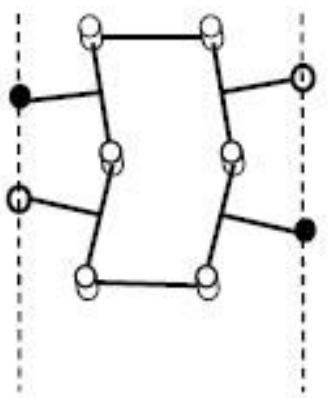

(b)

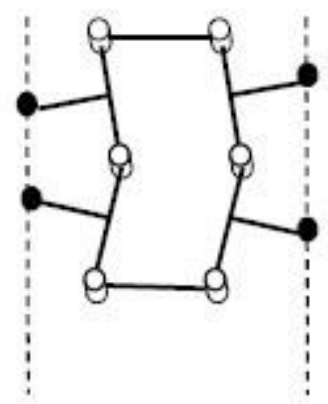

(c)

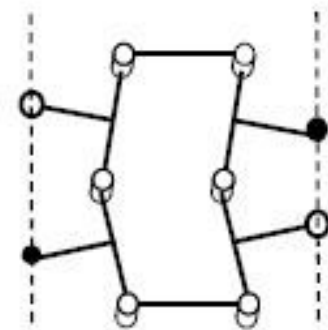

(d)

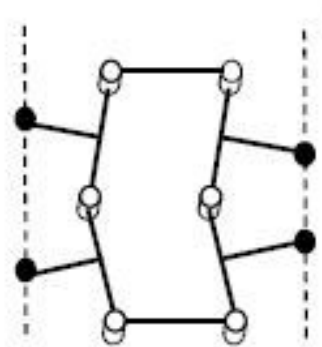

(e)

Figure 3

Diagram of tree climbing gait. (a) $t=0$. (b) $t=T / 4$. (c) $t=T / 2$. (d) $t=3 T / 4$. (e) $t=T$.

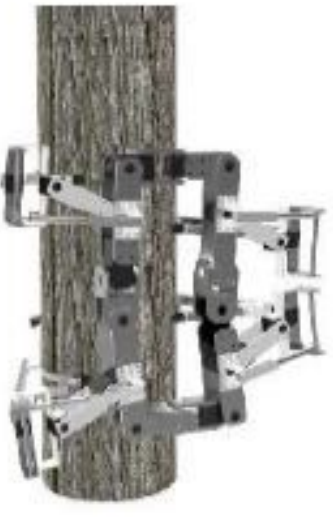

(a)

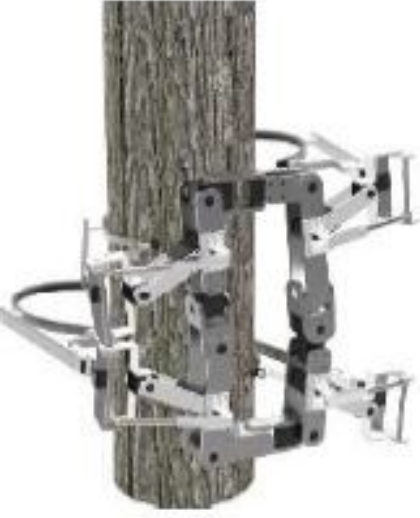

(b)

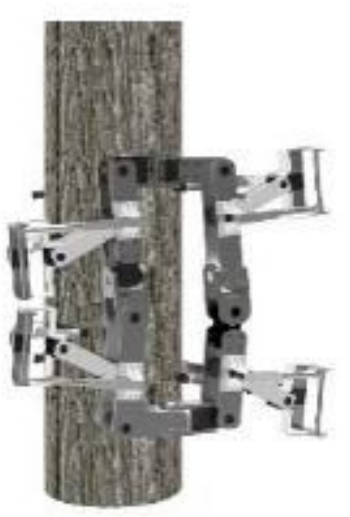

(c)

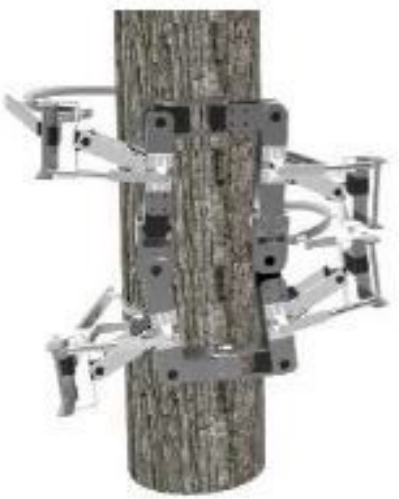

(d)

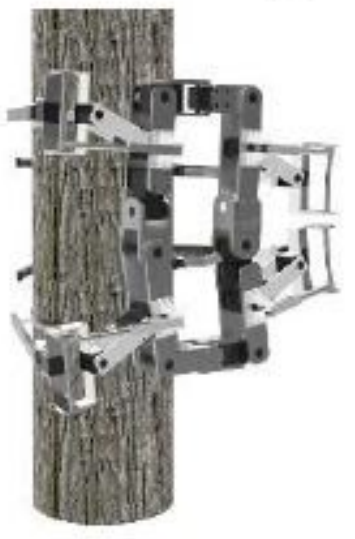

(e)

Figure 4 
Tree climbing robot model in subsequent working states. (a) $t=0$. (b) $t=T / 4$. (c) $t=T / 2$. (d) $t=3 T / 4$. (e) $t=T$.

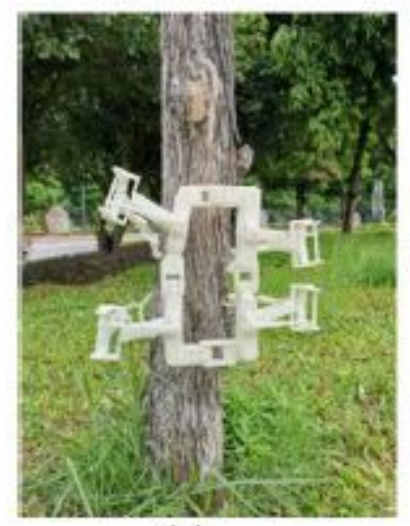

(a)

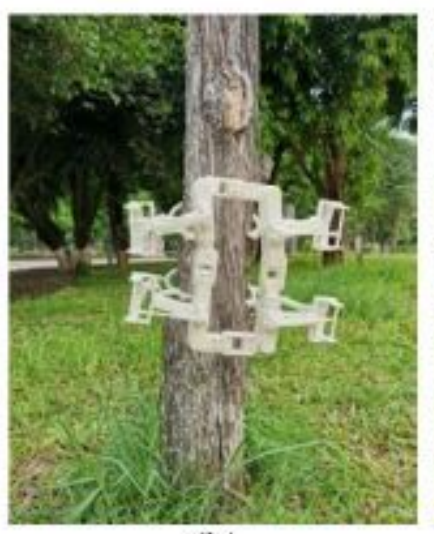

(b)

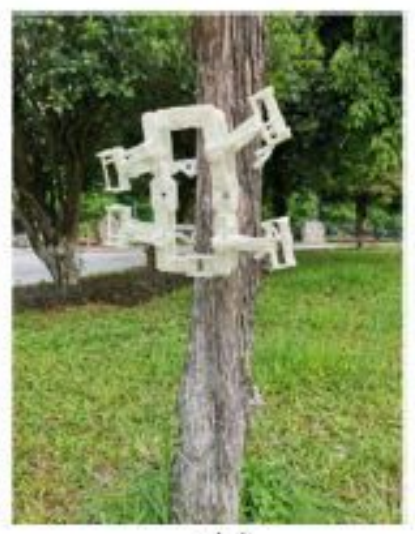

(c)

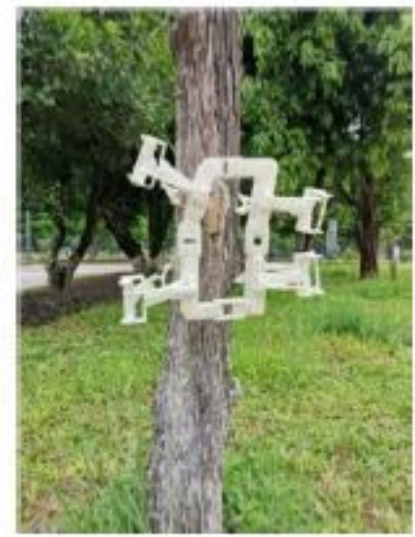

(d)

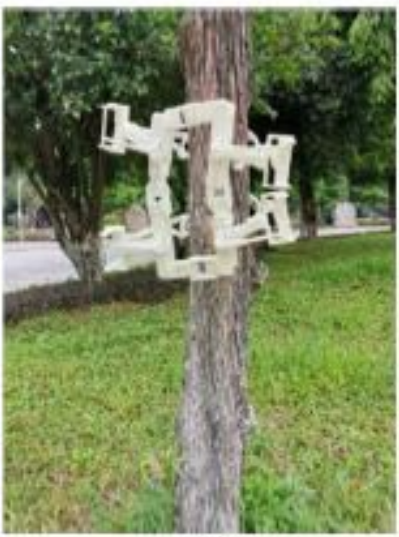

(e)

\section{Figure 5}

The prototype of the tree climbing robot mechanism: (a) the initial state. (b) Climbing the tree in T/2. (c) Back to initial state in T. (d) Climbing the tree in 3T/2. (e) Hug the trunk in $2 T$

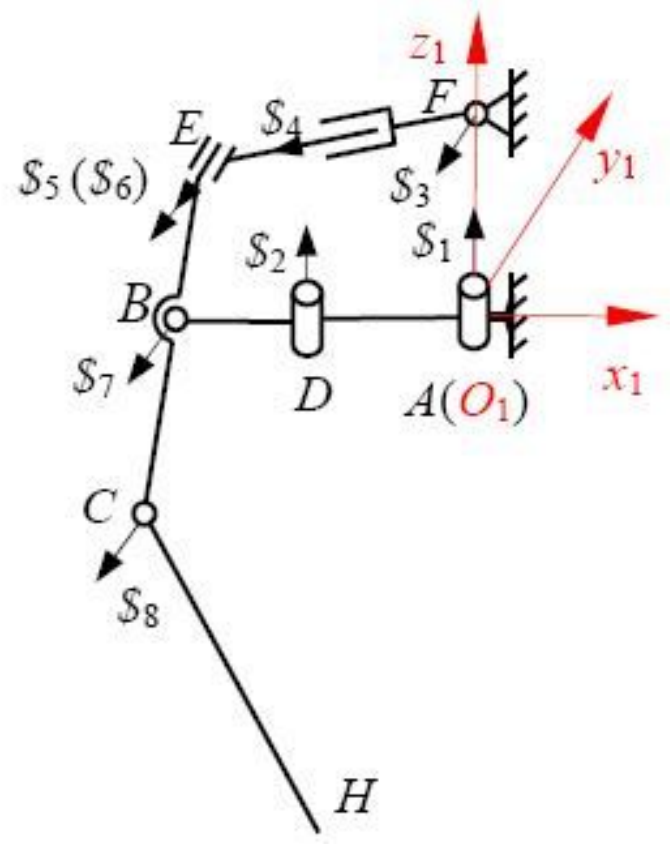


Figure 6

Leg mechanism link screw coordinate system

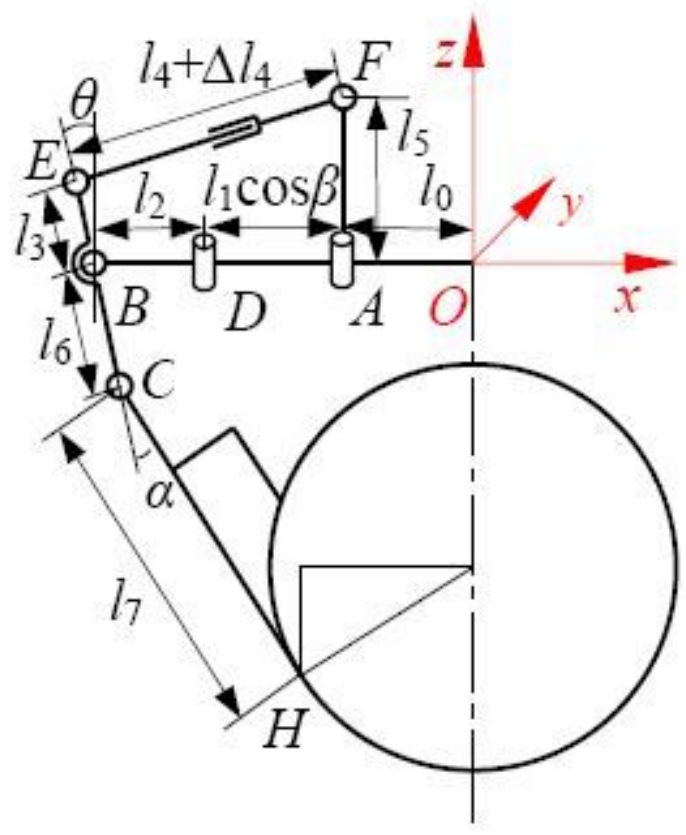

Figure 7

Leg mechanism coordinate system

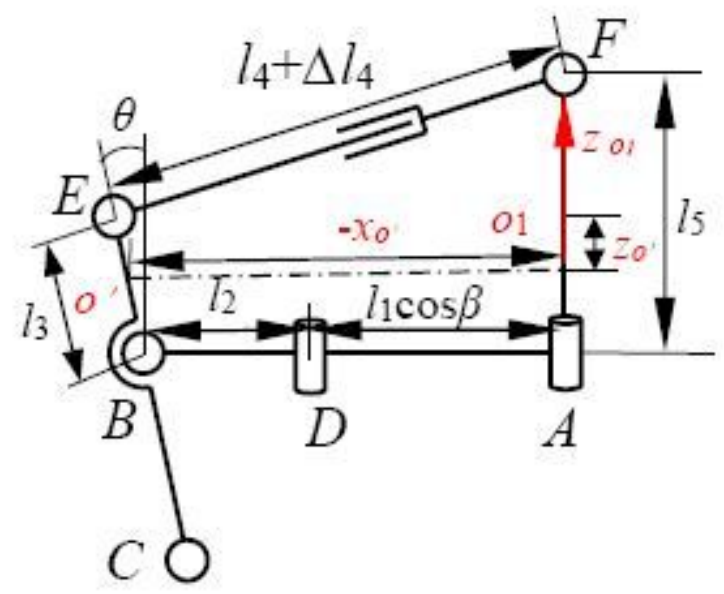

Figure 8

Projection diagram of parallel mechanism ABDEF 


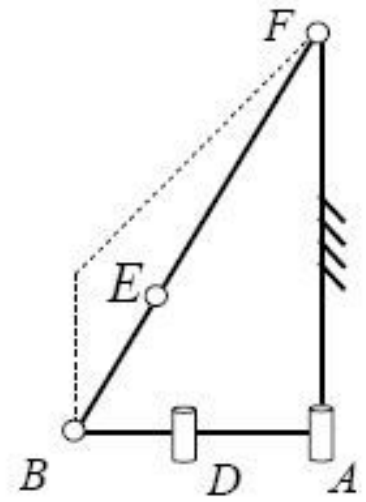

(a)

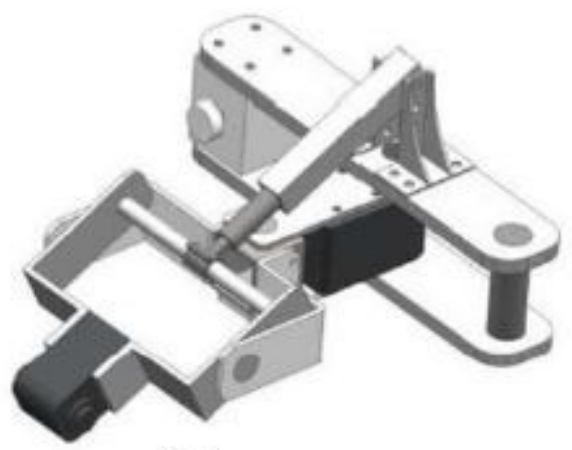

(b)

\section{Figure 9}

Singularity (I): The cases of the Eq. (23). (a) Diagram of the parallel mechanism. (b) Model of the parallel mechanism.



(a)

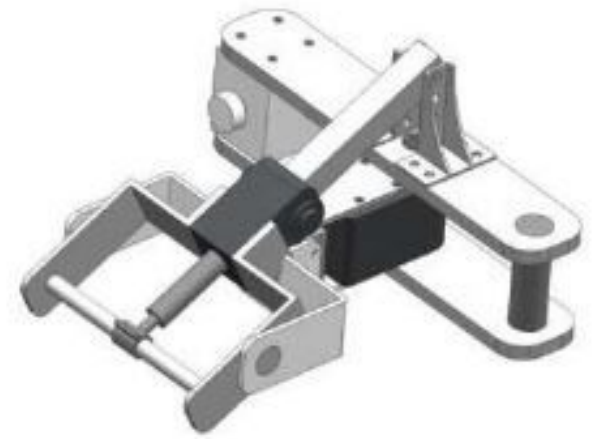

(b)

\section{Figure 10}

Singularity (II): The cases of the Eq. (24). (a) Diagram of the parallel mechanism. (b) Model of the parallel mechanism. 


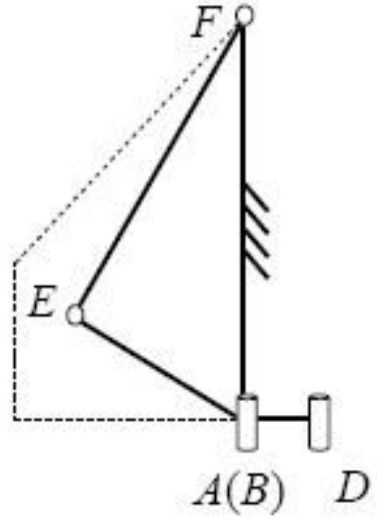

(a)

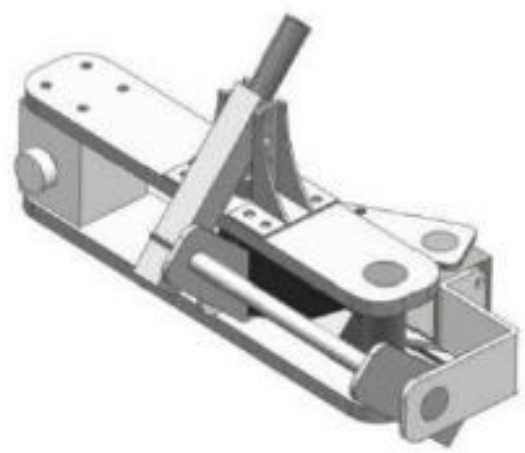

(b)

\section{Figure 11}

Singularity (II): The cases of the Eq. (25). (a) Diagram of the parallel mechanism. (b) Model of the parallel mechanism.

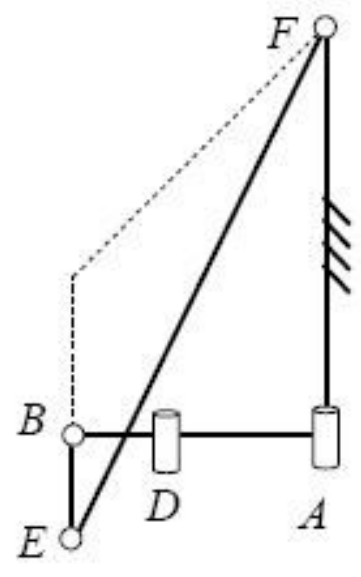

(a)

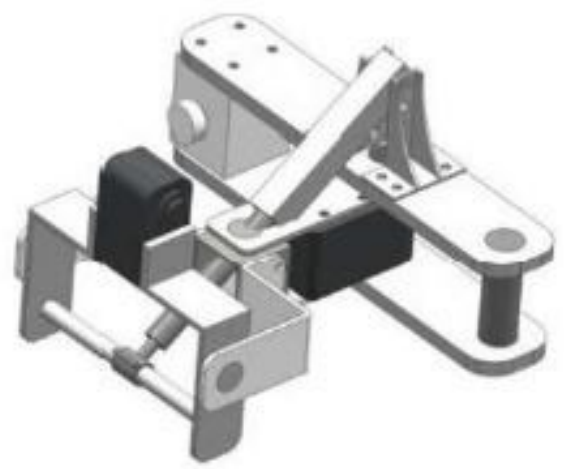

(b)

\section{Figure 12}

Singularity (III): The cases of the Eq. (26). (a) Diagram of the parallel mechanism. (b) Model of the parallel mechanism. 


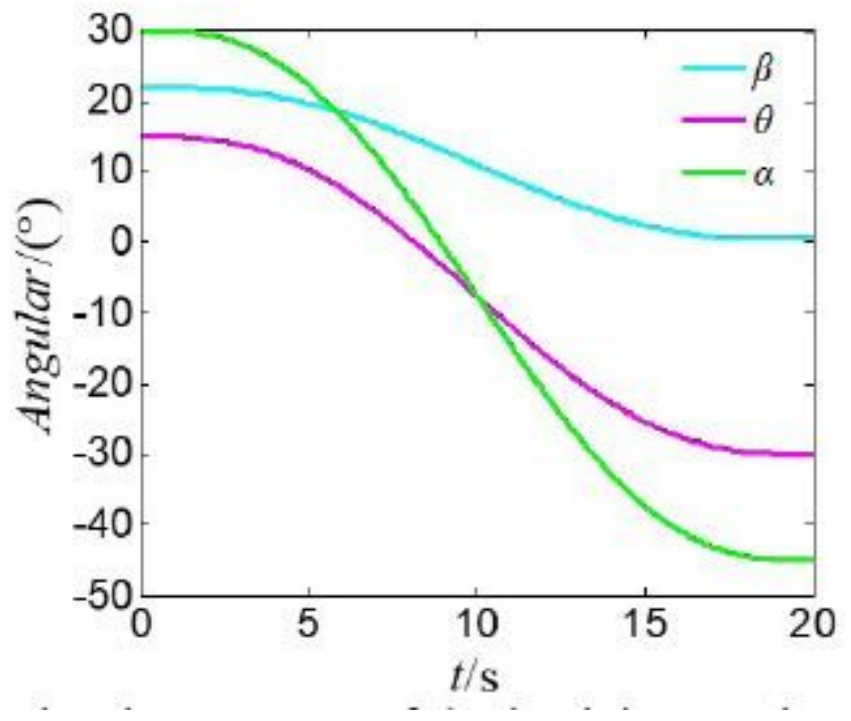

Figure 13

The change curve of the leg joint angular over a time period.

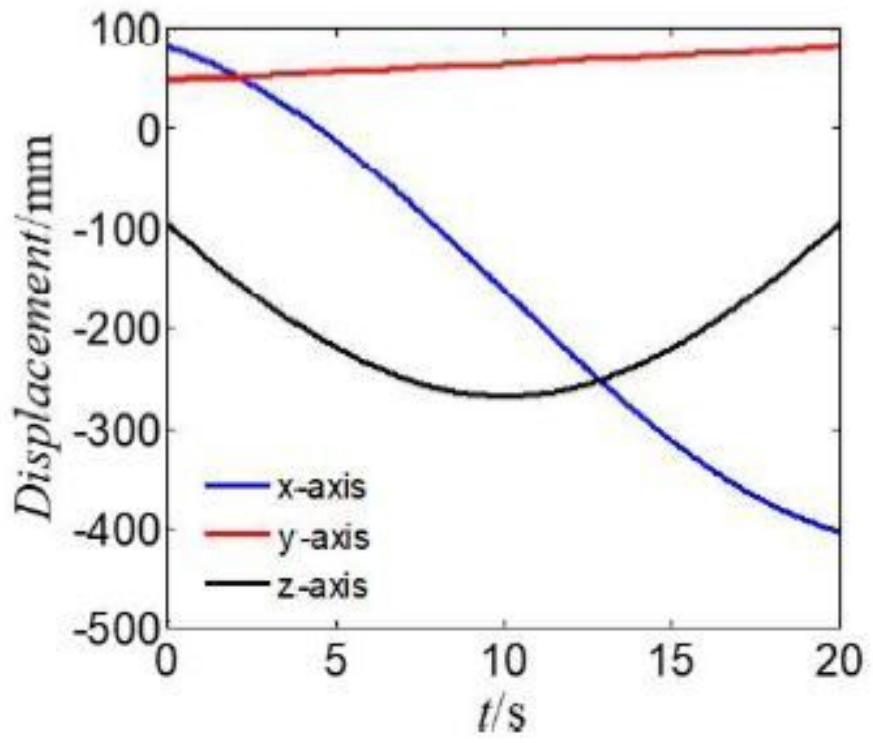

Figure 14

The change curve of the displacement over a time period. 


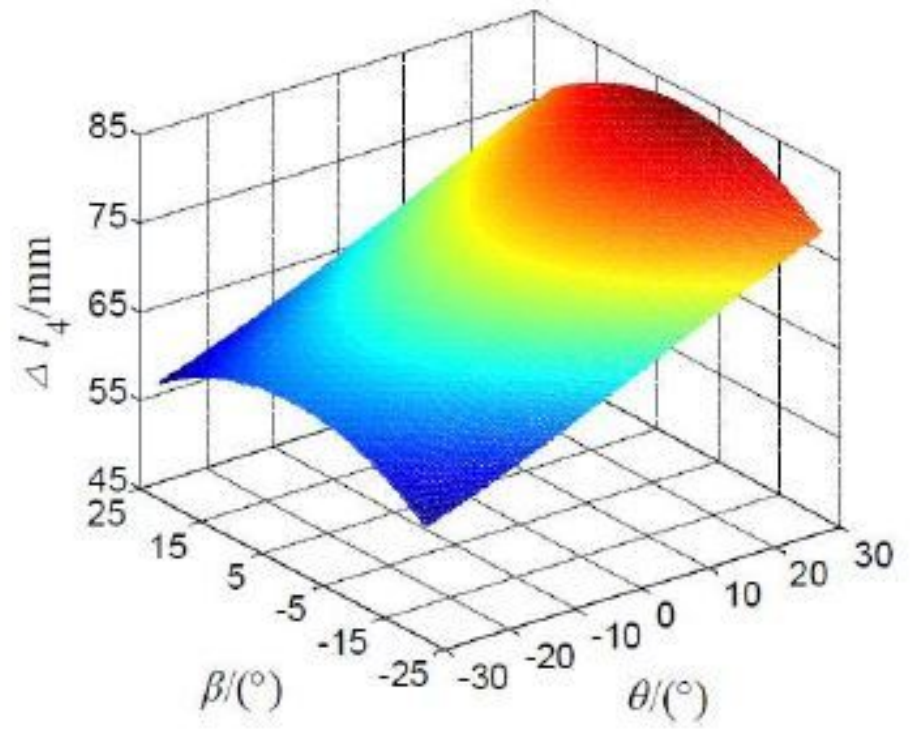

Figure 15

Relationship between the driving displacement $\Delta / 4$ and value range of angle $\beta$ and $\theta$. 